\title{
Biochemical and biomechanical properties of tendons in two commercial types of chickens
}

\author{
M. Moussa, R. Babilé, X. Fernandez and H. Rémignon ${ }^{\dagger}$ \\ INRA, Université de Toulouse, UMR1289, Tissus Animaux, Nutrition, Digestion, Ecosystème et Métabolisme, Chemin de Borde-Rouge-Auzeville, BP 52627, \\ F-31326 Castanet-Tolosan Cedex, France; INPT-ENSAT, F-31326 Castanet-Tolosan Cedex, France, ENVT, F-31076 Toulouse Cedex 3, France
}

(Received 28 September 2006; Accepted 2 April 2007)

The quality of the attachment of meat to bone is often reported to be insufficient by more and more poultry's consumers. This is particularly true for thigh meat in broilers. The aim of this study was to compare muscle to bone attachment (namely, tendons) from a biomechanical and a biochemical point of view in 50 standard (S) and 50 Label Rouge (LR) chickens. Carcasses weighted around $1.7 \mathrm{~kg}$ in the two groups. Two tendons were harvested and proceeded for passive stretch tests, prior to cooking or not, to determine main mechanical characteristics (maximum load, stiffness and longitudinal strain). Biochemical parameters such as dry matter percentage, total collagen content, collagen solubility and sulphated glycosaminoglycans (sGAGs) content were also determined. Results showed that biomechanical values differ largely between the two studied tendons. For a given tendon, the values were also different between the two groups of chickens mainly after cooking. The results clearly showed that, mainly after cooking, the mechanical resistance of tendon to stretch was better in $L R$ than in $S$ chickens. $L R$ chickens were reported to have tendons with higher collagen and sGAGs contents associated with a lower collagen solubility. These differences may explain biomechanical differences observed for the two types of tendons and could be due to increased age and/or higher physical activity of $L R$ chickens.

Keywords: broilers, collagen, maximum load, stiffness, sulphated glycosaminoglycans

\section{Introduction}

Tendons serve to attach soft tissue to bone and are specialised to resist and transmit the forces generated by muscle fibres to the skeletal system (Koob and Summers, 2002). Collagen is the major structural protein of the connective tissue and the most abundant protein of the tendon in which it is found in fibrous state (Birk et al., 1989). The collagen content is about $70 \%$ of the dry matter in the tendon (Nimni and Harkness, 1988). There are also certain non-collagenous proteins in tendon, such as osteopontin, osteocalcin and proteoglycans (PGs) (Grover and Shoshan, 1980). PGs consist of a core protein with one or more covalently attached glycosaminoglycans (GAGs). Theses GAGs chains are long-linear carbohydrate polymers that are negatively charged under physiological conditions due to the occurrence of sulphate and uronic acid group. Despite their small amount (less than $5 \%$ of total dry matter), GAGs could play an important role in the biomechanical properties of tendon. A previous study has shown that GAGs bind

† E-mail: remignon@ensat.fr the collagen fibril to another, like a molecular bridge, providing interfibrillar connections (Derwin et al., 2001). This architecture would suggest their possible role in influencing the mechanical integrity of the tendon structure. It has also been suggested that GAGs and associated PG molecules would be able to transmit forces from one collagen fibril to another (Redaelli et al., 2003).

Out of a pathological situation, factors such as age of the animal or physical activity can modify the biochemical and biomechanical properties of tendon (Hae Yoon et al., 2003). In connective tissues, the main change with ageing is the stiffening of collagen (Gal and Everitt, 1970). According to Voet and Voet (2005), ageing induces an increase in the degree of interaction between and within the elementary collagen fibrils. This makes the connective tissue become increasingly resistant to solubilisation (Butzow and Eichhorn, 1968). These changes in collagen quality and/or quantity could influence the mechanical properties of tendon as expected by Buchanan and Marsh (2002) who reported that changes in biomechanical properties of tendons are associated with changes in collagen concentration. The total content of GAGs mainly depends on the 
biomechanical role of tendons. Parry et al. (1982) report that, in the flexor digitorum profundus tendon of rabbit, the GAGs content vary from $2.3-3.5 \%$ to $0.2 \%$ of the dry weight on the pressure side and in tensional parts, respectively. Ageing is also normally associated with an increase in total GAGs content in tendons (Derwin et al., 2001).

In response to physical activities, tendons have the ability to modulate their structural, biochemical and biomechanical properties to meet specific requirements (Buchanan and Marsh, 2002). Under normal physiologic conditions, there is both static and dynamic tensile loading on collagen molecules and associated GAGs and PGs in tendons. An increase in tensile loading of the tendon will induce a mechanical-chemical transduction leading to modifications in these structural elements (Viidik et al., 1996). Woo et al. (1980) found an increase in total collagen content of digitorum extensor tendon in pigs with exercised tendons. On the other hand, Hae Yoon et al. (2003) reported that a moderate exercise led to a $20 \%$ increase in the total GAGs content in the tendon of chicken.

For consumers and meat processors, the quality of the attachment of muscle to bone can have a particular interest when meat needs to be harvested for processing or eating. This is of particular interest in small animals, such as poultry or rabbits, because whole anatomical parts are generally eaten. In France, fast-growing standard (S) broilers have a poor image because they are often reported to present, mainly in the thigh, a very poor cohesion between meat and bone especially after a safe (or long) time of cooking. On the other hand, more extensive poultry production systems, such as Label Rouge (LR) chickens are considered to have a higher quality of meat associated with a better cohesiveness. In the US, Alvarado et al. (2005) also reported that free-range chicken drumsticks had a stronger attachment to the bone than did the commercial chickens drumsticks.

Standard broiler (S) or Label Rouge (LR) chicken production systems differ in many ways such as genetic origin, feed composition, rearing density, etc. (for a review see Rémignon and Culioli, 1995). Among all these factors, the age at slaughtering ( 35 to 40 days v. 81 days old for $S$ and LR chickens, respectively) and the access to free-range only for $L R$ chickens may have a direct influence on the structure and development of various tendons. The aim of this study was to verify if those largely different conditions of rearing are sufficient to modify the biomechanical and biochemical properties of two different tendons harvested from S and LR chickens.

\section{Material and methods}

Animals

Fifty slow-growing chickens (LR type) and 50 broilers (S type) aged 82 and 41 days, respectively, were conventionally slaughtered in a poultry slaughterhouse and immediately stored at $+4^{\circ} \mathrm{C}$ for $24 \mathrm{~h}$. Within a day, all the carcasses were dissected and the two thigh-leg and breast-wing parts of the carcasses were weighed, vacuum packaged, frozen and then kept at $-20^{\circ} \mathrm{C}$ until analysis.

\section{Measurements of biomechanical properties}

After an overnight thawing at $4^{\circ} \mathrm{C}$, the gastrocnemius (Gst) and pectoralis minor $(\mathrm{Pm})$ tendons were precisely dissected and immediately immersed in physiological serum to avoid severe dehydration. The Gst tendon was identified as the one that links the $m$. gastrocnemius to the tarso-metatarsus while the $\mathrm{Pm}$ tendon relies the $m$. pectoralis minor to the humerus bone (Nickel et al., 1977). For each animal, one tendon was first used for performing biomechanical tests (tensile tests see above) and then frozen until biochemical analysis. Same measurements were also performed after cooking of the second tendon (previously vacuum packaged) in a water-bath at $80^{\circ} \mathrm{C}$ for $10 \mathrm{~min}$.

Passive stretch (or tensile) tests were performed with a universal testing machine (MTS System Corporation, Eden Prairie, MN, USA) driven by Testworks 4.0 software. Briefly, the two extremities of the tendons were first deeply frozen in liquid nitrogen to ensure a good adhesion and then inserted in two jaws. One jaw was fixed on the cross-head of the universal testing machine and could move vertically while the second jaw remained fixed on the basis of the machine. The speed of the cross-head was set to $50 \mathrm{~mm} / \mathrm{min}$ and the length and force values applied to the tendon were recorded (frequency $=100 \mathrm{~Hz}$ ) until the rupture of the tendon. From the data collected, the software allowed us to measure the following parameters: maximum load (in N), energy at maximum load (in $\mathrm{N} \cdot \mathrm{mm}$ ), stiffness value during the elastic elongation (in $\mathrm{N} / \mathrm{mm}$ ) and elongation (or $\Delta L$ in $\mathrm{mm}$ ) of the tendon at the maximum load. The initial length $\left(L_{0}\right.$ in $\mathrm{mm}$ ) of the tendon was recorded before the beginning of the test and the longitudinal strain was calculated as $\Delta L / L_{0}$.

Immediately after the tensile test, parts of the tendon were removed from the jaws, weighed (fresh weight in g), and then frozen and stored at $-20^{\circ} \mathrm{C}$ until biochemical analysis.

\section{Measurements of biochemical properties}

All the measurements were done in duplicate. Frozen samples were ground in a mortar. The dry matter content was determined at $103^{\circ} \mathrm{C}$ for $24 \mathrm{~h}$ according to Journal Officiel des Communautés Européennes (1971).

Determination of the total collagen content. The total collagen content was determined according to Woessner (1961). Briefly, total hydroxyproline (OH-Pro) content of the tissue was determined after total acidic hydrolysis and the $\mathrm{OH}$-Pro content spectrometrically determined at $557 \mathrm{~nm}$. The mean rate of $\mathrm{OH}$-Pro in collagen was estimated to be $14.6 \%$.

Determination of collagen solubility. The soluble fraction of the collagen was determined according to Snowden and Weidemann (1978). Samples were first heated in a buffer $\left(0.14 \mathrm{~mol} / / \mathrm{NaCl}, 0.01 \mathrm{~mol} / / \mathrm{PO}_{4}{ }^{2-}, \mathrm{pH}\right.$ 7) for $10 \mathrm{~min}$ at 
$85^{\circ} \mathrm{C}$. Then the fraction of collagen solubilised by heating was removed after centrifugation at $4000 \times \mathbf{g}$ for $30 \mathrm{~min}$. Pellets were then submitted to enzyme (Pronase $E$ from Sigma-Aldrich, St Louis, MD, USA; $1 \mathrm{mg} / \mathrm{ml}$ ) digestion for $16 \mathrm{~h}$ at $20^{\circ} \mathrm{C}$. The solubilised products were collected in supernatant after centrifugation at $80000 \times \mathbf{g}$ for $30 \mathrm{~min}$. $\mathrm{OH}$-Pro content in the different collected fractions was determined according to Woessner (1961). Solubility of collagen was expressed as the percentage of soluble collagen extracted from total collagen.

Determination of the sulphated glycosaminoglycans (sGAGs) content. Samples were first denatured by heating for $10 \mathrm{~min}$ at $85^{\circ} \mathrm{C}$ in a buffer $(0.1 \mathrm{~mol} / \mathrm{l}$ Tris-acetate, $10 \mathrm{mmol} / \mathrm{l}$ calcium acetate, $\mathrm{pH}$ 7.8). After centrifugation $(4000 \times \mathbf{g}$ for $10 \mathrm{~min})$ supernatants were discarded while pellets were submitted to enzymatic digestion (Pronase $\mathrm{E}$, Sigma, $8.33 \mathrm{mg} / \mathrm{ll}$ ) at $37^{\circ} \mathrm{C}$ for $72 \mathrm{~h}$ and under agitation. Supernatants were finally collected after centrifugation at $2000 \times \mathbf{g}$ for $10 \mathrm{~min}$ and stored at $-20^{\circ} \mathrm{C}$ until analysis. sGAGs content (i.e. chondroitin sulphates + dermatan sulphates + keratan sulphates + heparin sulphates) was determined according to the recommendations of the manufacturer (Biocolor Ltd, UK) of the Blyscan ${ }^{\mathrm{TM}}$ sGAGs assay. Percentage of sGAGs sulphated on an $\mathrm{N}$ or an 0 were also determined according to the manufacturer' procedures.

\section{Statistical analysis}

All the data compared values between the two groups of animals by using the general linear method (GLM) procedure of Statistical Analysis Systems Institute (2004). When appropriate, means were compared according to Duncan's test.

\section{Results}

As expected, animals from the two groups exhibited similar carcass (around $1700 \mathrm{~g}$ ) and thigh (approx. $325 \mathrm{~g}$ ) weights.

\section{Biomechanical results}

Biomechanical results recorded in Gst and Pm tendons are reported in Tables 1 and 2, respectively. Before cooking, neither maximum load nor stiffness nor energy at the maximum load values were found to be significantly different between LR and S chickens. On the contrary, longitudinal strain of Gst and Pm raw tendons were found to be higher in S than in LR birds. After cooking, only the longitudinal stress values were found to be similar in LR and $S$ chickens while other biomechanical parameters were significantly higher in LR than in $S$ chickens.

From these first observations, we can conclude that the two studied raw tendons have similar biomechanical behaviours in the two groups of animals. In consequence, this behaviour seems to be independent of the physiological function and particularly independent of the level of activity of the tendon in the live bird because it can be hypothesised that the Gst tendon is much more used than the Pm in actions like standing up or walking to reach feeders and drinkers. Moreover, $S$ broilers have a natural tendency to stay still for a long time (Bizeray et al., 2000), while LR chickens are usually described as more active.

For both types of birds, the two tendons were found to be less extensible at the raw state than after cooking. After the thermal denaturation induced by cooking, the absolute mechanical values were also less important than before. This thermal denaturation affected more the $S$ than the LR tendons as illustrated by maximum energy value reductions observed, for each tendon, between the raw and cooked state.

\section{Biochemical results}

Data reported in Table 3 showed that Gst tendons from the two types of birds weighed approximately the same, while $\mathrm{Pm}$ tendons were significantly heavier in $\mathrm{LR}$ than in $\mathrm{S}$ chickens. Dry matter content was more important in tendons from $L R$ than from $S$ birds, indicating more important biochemical content in older and more active birds. For all samples, total collagen content represented more than

Table 1 Biomechanical values in raw or cooked Gst tendons from $S$ and $L R$ chickens (values are means $\pm s . d$. $(\mathrm{n}=50)$ )

\begin{tabular}{|c|c|c|c|c|c|}
\hline Variables & Treatment & LR & $S$ & $\mathrm{~F}$ & Significance $^{\dagger}$ \\
\hline \multirow[t]{2}{*}{ Maximum load (N) } & Raw & $130.9 \pm 45.5$ & $128.7 \pm 30.3$ & 0.04 & NS \\
\hline & Cooked & $25.7 \pm 10.7$ & $14.9 \pm 7.6$ & 30.37 & $* * *$ \\
\hline \multirow[t]{2}{*}{ Stiffness (N/mm) } & Raw & $26.7 \pm 10.0$ & $23.5 \pm 6.1$ & 1.25 & NS \\
\hline & Cooked & $7.1 \pm 3.0$ & $4.1 \pm 1.7$ & 37.55 & $* * *$ \\
\hline \multirow[t]{2}{*}{ Maximum energy $(\mathrm{N} \cdot \mathrm{mm})$} & Raw & $677.6 \pm 605.5$ & $633.6 \pm 386.5$ & 0.71 & NS \\
\hline & Cooked & $62.0 \pm 28.1$ & $37.3 \pm 13.0$ & 20.92 & *** \\
\hline \multirow[t]{2}{*}{ Longitudinal strain (at maximum load) } & Raw & $0.26 \pm 0.17$ & $0.38 \pm 0.21$ & 4.14 & * \\
\hline & Cooked & $0.72 \pm 0.37$ & $0.61 \pm 0.42$ & 0.86 & NS \\
\hline
\end{tabular}

Abbreviations are: Gst = gastrocnemius; $\mathrm{LR}=$ Label Rouge; NS = non-significant; $\mathrm{S}=$ standard; s.d. = standard deviation .

'Significance levels: ${ }^{*} P<0.05,{ }^{*} P<0.01,{ }^{* *} P<0.001$. 
Moussa, Babilé, Fernandez and Rémignon

Table 2 Biomechanical values in raw or cooked Pm tendons from $S$ and $L R$ chickens (values are means \pm s.d. $(\mathrm{n}=50)$ )

\begin{tabular}{llcccc}
\hline \hline Variables & Treatment & LR & S & F & Significance $^{+}$ \\
\hline Maximum load (N) & Raw & $116.7 \pm 33.0$ & $115.7 \pm 26.6$ & 4.52 & NS \\
& Cooked & $66.0 \pm 23.1$ & $46.5 \pm 15.8$ & 15.15 & $* * *$ \\
Stiffness (N/mm) & Raw & $28.6 \pm 10.6$ & $25.5 \pm 6.9$ & 1.19 & NS \\
& Cooked & $11.1 \pm 4.0$ & $9.1 \pm 2.8$ & 6.93 & $*$ \\
Maximum energy (N. mm) & Raw & $396.1 \pm 203.2$ & $371.1 \pm 126.7$ & 3.65 & NS \\
& Cooked & $233.5 \pm 117.2$ & $130.9 \pm 72.1$ & 21.09 & $* * *$ \\
Longitudinal strain (at maximum load) & Raw & $0.39 \pm 0.15$ & $0.77 \pm 0.31$ & 25.7 & $* * *$ \\
& Cooked & $3.40 \pm 2.89$ & $3.42 \pm 4.42$ & 0.00 & NS \\
\hline \hline
\end{tabular}

Abbreviations are: $\mathrm{Gst}=$ gastrocnemius; $\mathrm{LR}=$ Label Rouge; NS = non-significant; $\mathrm{Pm}=$ pectoralis minor, $\mathrm{S}=$ standard; $\mathrm{s}$.d. = standard deviation.

'See Table 1 for significance levels.

Table 3 Biochemical parameters in Gst and Pm tendons from S and LR chickens (values are means \pm s.d. $(\mathrm{n}=50)$ )

\begin{tabular}{|c|c|c|c|c|c|}
\hline Variables & Tendon & LR & $S$ & $\mathrm{~F}$ & Significance $^{+}$ \\
\hline \multirow[t]{2}{*}{ Fresh weight (g) } & Gst & $0.73 \pm 0.1$ & $0.74 \pm 0.1$ & 0.28 & NS \\
\hline & $\mathrm{Pm}$ & $0.52 \pm 0.1$ & $0.34 \pm 0.1$ & 96.69 & $* * *$ \\
\hline \multirow[t]{2}{*}{$\%$ dry matter (DM) } & Gst & $33.0 \pm 2.4$ & $26.1 \pm 2.2$ & 166.21 & *** \\
\hline & $\mathrm{Pm}$ & $31.0 \pm 2.8$ & $29.5 \pm 2.6$ & 7.36 & ** \\
\hline \multirow[t]{2}{*}{ Total collagen (\% of DM) } & Gst & $73.7 \pm 7.8$ & $65.1 \pm 6.6$ & 40.14 & $* * *$ \\
\hline & $\mathrm{Pm}$ & $76.6 \pm 5.9$ & $71.4 \pm 5.4$ & 7.53 & ** \\
\hline \multirow[t]{2}{*}{ Solubility of collagen (\%) } & Gst & $67.6 \pm 12.3$ & $76.1 \pm 12.4$ & 11.69 & $* * *$ \\
\hline & $\mathrm{Pm}$ & $68.4 \pm 12.0$ & $73.4 \pm 9.6$ & 5.1 & * \\
\hline
\end{tabular}

Abbreviations are: Gst = gastrocnemius; $\mathrm{LR}=$ Label Rouge; NS = non-significant; $\mathrm{S}=$ standard; s.d. = standard deviation; Pm = pectoralis minor.

${ }^{\dagger}$ See Table 1 for significance levels.

two-thirds of the total dry matter content and was consequently the first biochemical component of the tendon in the chicken. The total collagen content was always higher in samples from $L R$ than from $S$ chickens whatever the tendon we considered. On the contrary, the solubility of collagen was less important in LR than in $S$ samples either for Gst or Pm tendons. According to Redaelli et al. (2003), the older age of LR birds could explain the observed differences in total collagen and collagen solubility. Nevertheless, increased physical activity of LR birds could also influence those parameters and then explain the lower differences observed in $\mathrm{Pm}$ than in Gst tendons which are much more involved in standing up, laying or walking.

Data reported in Figure 1 showed that Pm tendons contained two times less sGAG than Gst tendons. Moreover, only Gst tendons contained significantly more SGAG in LR than in $\mathrm{S}$ birds. This difference was not present in Pm tendons indicating that this parameter could be influenced by the type of tendon. In Figure 2 are reported the percentage of N-sulphated GAGs which are significantly higher in Gst tendons from LR than from $S$ chickens. This difference in percentage of the types of the sulphatation of the GAGs was not observed in Pm tendons from the two types of birds.

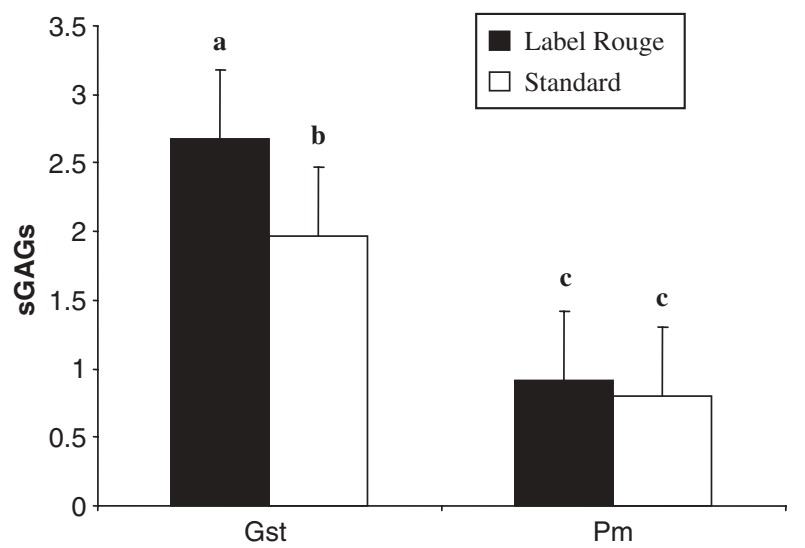

Figure 1 Total sulphated glycosaminoglycans (sGAGs) content (\% of dry matter) in the two studied tendons (gastrocnemius (Gst) and pectoralis minor ( $(\mathrm{Pm})$ ) from the two types of chickens (Label Rouge and standard). Values are means \pm s.d., $n=50$. Values with different letters are significantly different $(P<0.05)$.

\section{Discussion}

According to Buchanan and Marsh (2002), one might expect changes in biomechanical properties of tendons to be associated with changes in collagen concentration. This is not the case in our study since a significant difference in 


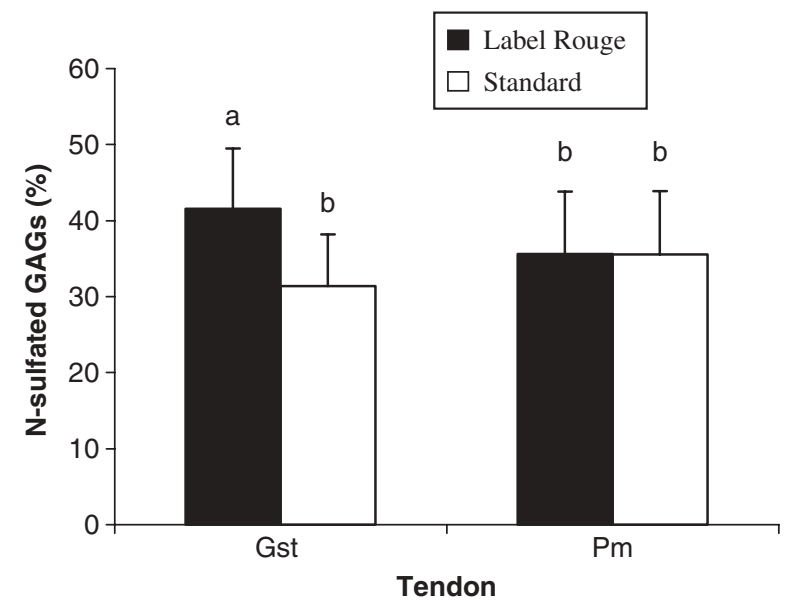

Figure 2 Percentages of $\mathrm{N}$-sulphated glucosaminoglycans (\% of total sGAGs content) in two studied tendons (gastrocnemius (Gst) and pectoralis minor $(\mathrm{Pm})$ ) from the two types of chickens (Label Rouge and standard). Values are means \pm s.d., $n=50$. Values with different letters are significantly different $(P<0.05)$.

total collagen content exists between the two groups of birds but it is not associated with biomechanical modifications. Many other studies also do not support this hypothesis: Viidik (1967) in rabbits, Vailas et al. (1985) in rats or Curwin et al. (1988) in chickens and Woo et al. (1981) in pigs.

As reviewed by Buchanan and Marsh (2002), it is generally admitted that ageing and training result in increased tensile strength and stiffness values in tendons. With increasing age, tendons are supposed to be more resistant to tensile strength because they have to support a heavier load due to an increase in body weight. In the present experiment, if the two groups of animals were killed at largely different ages (82 v. 40 days of age for LR and S groups, respectively), they exhibited same body and thigh weights. Previous studies also indicate that body compositions are approximately equivalent at these ages between the two types of birds. This indicates that the different tendons from the two groups of animals have to support the same loads. In consequence, it seems normal that most of the biomechanical parameters considered here were found to be in the same ranges for the two types of birds. Nevertheless, for raw tendons, the longitudinal strain value at maximum load was higher in $S$ than in LR samples. This parameter indicates that the degree of stretching was higher in S than in LR tendons. This could have been due to a lower degree of cross-linking of the molecule of collagen generally observed in younger birds and illustrated here by the higher solubility of collagen determined in tendons from $S$ animals.

After cooking, collagen content of the tendons is supposed to be the same as before because heating liberates less than $1 \%$ of the total collagen (data not shown). On the contrary, heating is able to modify proteins, such as collagen, by disrupting cross-links within the molecule. This may explain the dramatic decrease of biomechanical values after cooking for both types of birds. In cooked tendons, higher biomechanical values observed in LR birds indicate that they are better able to resist heat denaturation probably because of a higher level of collagen inter-molecular cross-linking normally associated with older age and higher level of physical activity (Sinex, 1964; Davison and Patel, 1975; Mitchell and Rigby, 1975).

If different degrees of cross-linking of the molecule of collagen can explain different longitudinal strain values and different resistance of the tendon to heat denaturation, other biochemical components may also be involved in the determination of mechanical values of the tendon. Cooking implies biochemical modifications, within the tissue, that helps explain the observed dramatic drop of biomechanical properties probably due to a severe dehydration of the tissue. In connective tissue, GAGs produce a genuine hydrophilic gel. This ability to bind water is directly due to the structure of the GAGs in which long filaments are made of repetitive disaccharide monomers particularly prone to form hydrogen bonds able to hold back surrounding water molecules (Neuzil and Valdiguié, 2004). In consequence, GAGs are considered as important regulators of tissue visco-elasticity. GAGs polysaccharide chains also tie together collagen fibrils in animal extracellular matrices and their increased number could be associated with increased resistance to stress load (Redaelli et al., 2003). Nevertheless, in the present study, an increase in the amount of sGAGs in the Gst tendon from the $L$ birds is not associated with an increase in corresponding biomechanical values in raw tendons. On the contrary, during cooking, the presence of higher sGAGs content in Gst tendons from the LR birds could have protected them from a severe dehydration and, consequently, given them a better resistance during the following biomechanical tests. Then, it can be hypothesised that the increased rate in sGAGs content was not sufficient to modify native biomechanical properties of the tendon but give to it a better resistance to cooking dehydration, leading to a better final conservation of native visco-elastic properties and biomechanical properties. This also explains why decreases of biomechanical values are less important in LR than in S animals after cooking. These differences in biochemical organisation of tendons could be due to the age of the animals, but also due to their physical activity because large differences in total SGAGs content are found between Gst and Pm tendons.

From a chemical point of view, GAGs can be sulphated at an $\mathrm{O}$ or an $\mathrm{N}$ atom. Among the seven existing sGAGs, heparan sulphate is the only one which is sulphated both on $\mathrm{O}$ and $\mathrm{N}$ atoms while others are only sulphated on $\mathrm{O}$ atoms. From the present results that show differences in the type of sulphatation of GAGs in Gst tendons, we must expect that different ways of rearing chickens are able to generate fine regulation of the metabolism of sGAGs. This could be one of the adaptative answers to increased solicitations of tendons. Because GAGs are only a small part of $P G s$, a precise study of these supramolecules could also give interesting information on possible modifications of 
the organisation of tendons in birds. Because of the close relationships between PGs (mainly decorin) and the collagen molecule in tendons, other biochemical implications might also be considered.

\section{Conclusions}

In this study we demonstrated that biomechanical properties of tendons from LR and $S$ chickens are different mainly after cooking. The low values recorded in $S$ chickens might explain the poor adherence of meat to bone generally reported in modern broilers. From a biochemical point of view, it seems that the most important differences concern the solubility of the collagen as well as the sGAGs and collagen contents. These modifications, mainly the sGAGs content variations, can explain the different water-holding capacity of tendons and therefore different resistance to heat dehydration leading to different mechanical resistances after cooking. Nevertheless, this experimental design failed to distinguish the relative effects of age and physical activity on the determination of biochemical and biomechanical properties of tendons in chickens. Further experiments are being conducted to determine if one of these two parameters has a particular impact on the organisation of the tendons in chickens.

\section{Acknowledgement}

The authors would like to thank $\mathrm{Dr}$ P. Swider (Purpan Hospital in Toulouse, France) for his help in biomechanical measurements.

\section{References}

Alvarado CZ, Wenger E and O'Keefe SF 2005. Consumer perceptions of meat quality and shelf-life in commercially raised broilers compared to organic free range broilers. Proceedings of the XVII European symposium on the quality of poultry meat, Doorwerth, The Netherlands, pp. 257-261.

Birk DE, Southern JF, Zycband El, Fallon JT and Trelstad RL 1989. Collagen fibril bundles: a branching assembly unit in tendon morphogenesis. Development 107, 437-443.

Bizeray D, Leterrier C, Constantin P, Picard M and Faure JM 2000. Early locomotor behaviour in genetic stocks of chickens with different growth rates. Applied Animal Behaviour Science 68, 231-242.

Buchanan $\mathrm{Cl}$ and Marsh RL 2002. Effects of exercise on the biomechanical, biochemical and structural properties of tendons. Comparative Biochemistry and Physiology 133, 1101-1107.

Butzow JJ and Eichhorn GL 1968. Physical chemical studies on the age changes in rat tail tendon collagen. Biochimica et Biophysica Acta 154, 208-219.

Curwin SL, Vailas AC and Wood J 1988. Immature tendon adaptation to strenuous exercise. Journal of Applied Physiology 65, 2297-2301.

Davison PF and Patel A 1975. Age-related changes in aldehyde location on rat tail tendon collagen. Biochemical and Biophysical Research Communications 65, 983-989.
Derwin KA, Soslowsky LJ, Kimura JH and Plaas AH 2001. Proteoglycans and glycosaminoglycan fine structure in the mouse tail tendon fascicle. Journal of Orthopaedic Research 19, 269-277.

Gal A and Everitt AV 1970. Age changes in the polymer composition of acid soluble collagen prepared from rat tail tendon. Experimental Gerontology 5, $1-5$.

Grover NB and Shoshan S 1980. Three-dimensional organization of collagen fibres in tendon. Tissue and Cell 12, 523-528.

Hae Yoon J, Brooks R, Kim YH, Terada M and Halper J 2003. Proteoglycans in chicken gastrocnemius tendons changes with exercise. Archives of Biochemistry and Biophysics 412, 279-286.

Journal Officiel des Communautés Européennes 1971. Dosage de I'humidité. Official Journal of the European Community L279, 8-11.

Koob TJ and Summers AP 2002. Tendon - bridging the gap. Comparative Biochemistry and Physiology 133, 905-909.

Mitchell TW and Rigby BJ 1975. In vivo and in vitro aging of collagen using an isometric melting technique. Biochimica et Biophysica Acta 393, 531-541.

Neuzil E and Valdiguié $P$ 2004. Biochemistry of glycosaminoglycans. International Movement Newsletter 2, 1-3.

Nickel R, Schummer A and Seiferle E 1977. Anatomy of the domestic birds. Verlag Paul Parey, Berlin-Hamburg.

Nimni ME and Harkness RD 1988. Molecular structures and functions of collagen. In Collagen biochemistry, vol. 1 (ed. ME Nimni), pp. 1-78. CRP Press, Boca Raton, FL.

Parry AD, Flint MH, Gillard GC and Craig AS 1982. A role for glycosaminoglycans in the development of collagen fibrils. FEBS Letters 149, 1-7. Redaelli A, Vesentini S, Soncini M, Vena P, Mantero S and Montevecchi FM 2003. Possible role of decorin glycosaminoglycans in fibril to fibril force transfer in relative mature tendons - a computational study from molecular to microstructural level. Journal of Biomechanics 36, 1555-1569.

Rémignon $\mathrm{H}$ and Culioli J 1995. Meat quality traits of French 'Label' chickens In Proceedings of the XII European symposium on the quality of poultry meat (ed. R Cepero Briz), pp. 145-150. Spanish Branch of the World Poultry Science Association, Zaragoza, Spain.

Sinex FM 1964. Cross-linkage and aging. Advanced in Gerontological Research 18, 165-180.

Snowden JMcK and Weidemann JF 1978. A morphological and biochemical examination of the hydrothermal denaturation of collagen. Meat Science 2, $1-18$.

Statistical Analysis Systems Institute 2004. SAS/STAT 9.1. SAS Institute Inc., Cary, NC, USA.

Vailas AC, Pedrini VA, Pedrini-Mille A and Holloszy J0 1985. Patellar tendon matrix changes associated with aging and voluntary exercise. Journal of Applied Physiology 58, 1572-1576.

Viidik A 1967. The effect of training on the tensile strength of isolated rabbit tendons. Scandinavian Journal of Plastic and Reconstructive Surgery 1, 141-147.

Viidik A, Nielsen HM and Skalicky M 1996. Influence of physical exercise on aging rats: II. Life-long exercise delays aging of tail tendon collagen. Mechanisms of Ageing and Development 88, 139-148.

Voet D and Voet JG 2005. Biochimie, 2nd edition. De Boeck University, Brussels.

Woessner Jr JF 1961. The determination of hydroxyproline in tissue and protein samples containing small portions of the amino acid. Archives of Biochemistry and Biophysics 93, 440-447.

Woo SL, Ritter MA and Amiel D 1980. The biomechanical and biochemical properties of swine tendon: long-term effects of exercise on the digital extensors. Connective Tissue Research 7, 177-183.

Woo SL, Gomez MA, Amiel D, Ritter MA, Gelberman RH and Akeson WH 1981. The effects of exercise on the biomechanical and biochemical properties of swine digital flexor tendons. Journal of Biomechanical Engineering 103, 51-56. 